Case Report

\title{
Hydrocephalus and Spasticity in Motor Area AVM, a Case Report
}

\author{
Veranis Sotiris ${ }^{1,}$, , Papageorgiou Demetrios ${ }^{1}$, Mpazinas Theodoros ${ }^{2}$, Lagios Konstantinos ${ }^{2}$ \\ ${ }^{1}$ Neurosurgical Department, 251 General Air Force and Reserve Hospital, Athens, Greece \\ ${ }^{2}$ Neurointernvantional Unit, 251 General Air Force and Reserve Hospital, Athens, Greece \\ Email address: \\ veranissotiris@gmail.com (V. Sotiris) \\ ${ }^{*}$ Corresponding author
}

To cite this article:

Veranis Sotiris, Papageorgiou Demetrios, Mpazinas Theodoros, Lagios Konstantinos. Hydrocephalus and Spasticity in Motor Area AVM, a Case Report. Clinical Neurology and Neuroscience. Vol. 3, No. 1, 2019, pp. 6-10. doi: 10.11648/j.cnn.20190301.12

Received: February 25, 2019; Accepted: April 8, 2019; Published: May 15, 2019

\begin{abstract}
Obstructive hydrocephalus is a rare complication of brain AVM's. It usually diagnosed because of posterior fossa AVM's. This article is about a case of a 40-year-old female patient suffering from a right fronto-parietal unruptured grade IV AVM located in the central sulcus who developed obstructive hydrocephalus and worsening of left sided weakness and spasticity. The AVM has been embolized twice in the past and no further treatment was offered because of the adjacent motor cortex and concerns of permanent neurological damage. Patient was treated with navigated ventricular-peritoneal shunt with a programmable valve. Interestingly there was an improvement of spasticity and weakness.
\end{abstract}

Keywords: Hydrocephalus, Arteriovenous Malformation, Spasticity, Motor Cortex

\section{Introduction}

Obstructive hydrocephalus because of dilated internal cerebral veins is a rare complication [1]. Motor cortex arteriovenous malformations are known to cause spasticity and hemiparesis on the contralateral side of the body. [2] Obstructive hydrocephalus because of dilated internal cerebral veins is a rare complication. Venous hypertension and obstructive hydrocephalus could both reduce the cerebral blood flow. [9] Brain cortex near the AVM nidus is possibly affected more than remote cortex. [5].

Brain parenchyma around an arteriovenous malformation is depleted of cerebral blood flow because of a flow stealing phenomenon caused by the AVM [16].

Draining veins of arteriovenous malformation are becoming dilated and thickened and resemble more the arterial than the venous wall. This process is known as the arterialisation of the cerebral veins caused by the abnormal arteriovenous communication. [6] Thick and enlarged vessels could possibly act as a space occupying lesion.

\section{Method}

This case is about a 40 years old right handed female patient with a history of a right fronto-parietal AVM grade IV. The AVM was diagnosed ten years ago while investigating numbness of the left arm with brain CT and MRI. There had been two attempts ( 8 and 5 years ago) to partially embolize the arteriovenous malformation in another institution [3]. According to patient clinical history the second attempt to embolize the AVM was complicated with left hemiparesis which resolved 6 months later under physical rehabilitation. [13] She presented to our clinic with a 6 months' history of worsening hemiparesis and spasticity on the left side of her body. Modified Ashworth scale was used for evaluating spasticity, (elbow 3, wrist 3, finger 3, thumb 3, hamstring 2, quadriceps 3, gastrocnemius 2, soleus 2). Patient was unable to walk and upper and lower limb strength was $-2 / 5$ and $-3 / 5$ respectively. Neuroimaging studies with MRI (Figure 3), CTA (Figures 1,2) and DSA depicted no alterations of the partially emoblized AVM nidus. Furthermore, obstructive hydrocephalus caused by dilated internal cerebral veins at the 
level of Monroe foramen, was diagnosed. Patient was treated with a ventriculoperitoneal shunt and evaluated postoperatively, 1 month and at 3 month follow up examination. Third ventriculostomy was not an option in our case because of the dilated veins at the level of Monroe foramen. We concluded that there was a significant unacceptable risk of vein injury and bleeding by attempting to pass the ventriculoscope near these dilated veins. [11, 4]

Ventricular catheter was inserted using neuronavigation (Stealth Medtronic) through a left frontal burr hole [15]. Placement of the ventricular catheter into the lateral ventricles is navigated mainly because of narrow ventricles in idiopathic intracranial hypertension [8]. Neuronavigation (Figure 4) was used to choose with accuracy the placement of the burrhole and corticotomy in respect of dilated cortical veins, because of venous hypertension. In addition to the length of the catheter inserted was accurately adjusted not to interfere with the enlarged veins adjacent to foramen of Monroe [14]. Ventricular catheter was connected with a programmable Codman-Certas Plus Valve with antisiphon device adjusted to 5 (opening pressure $145 \mathrm{mmH} 2 \mathrm{O}$ ). There were not complications of the procedure and patient mobilized from the first postoperative day. A programmable shunt was chosen and the valve adjusted from 5 to 6 (opening pressure $180 \mathrm{mmH} 20$ ) in the $4^{\text {th }}$ postoperative day because patient depicted symptoms of endocranial hypotension. [10] After valve setting correction patient improved [12].

A postoperative head CT scan depicted accurate placement of the ventricular catheter and shrinkage of the ventricles and patient discharged to physiotherapy.

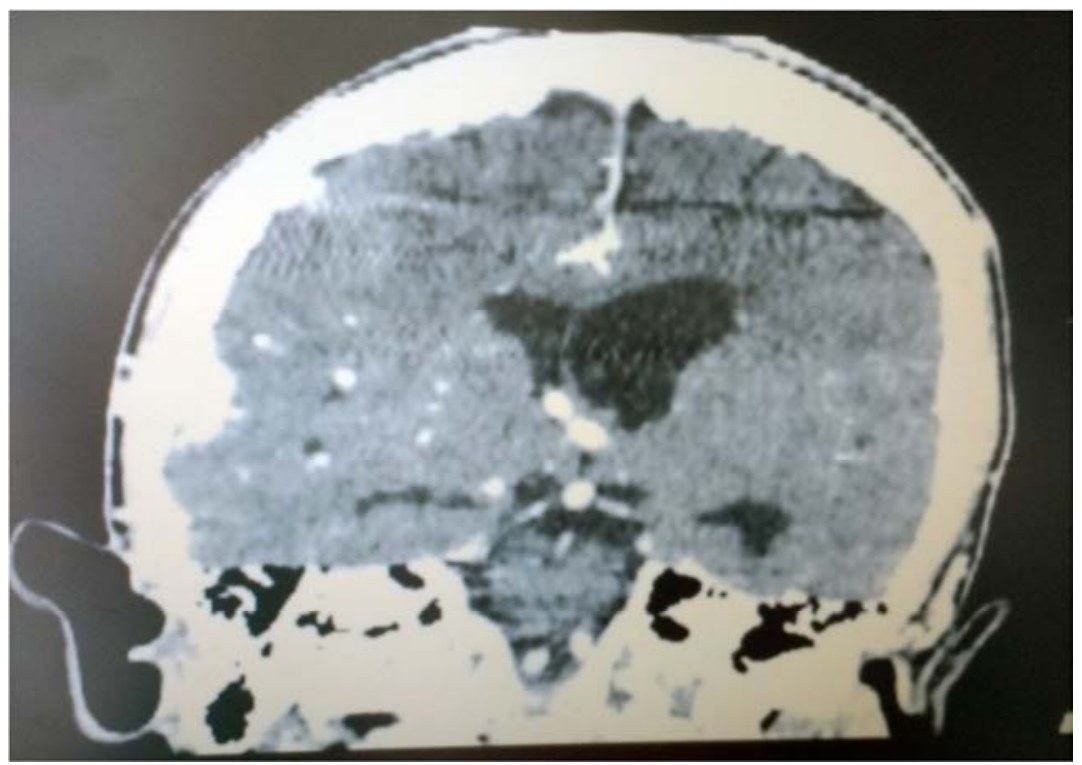

Figure 1. Preoperative coronal CT scan depicting obstructive hydrocephalus because of dilated internal cerebral veins.

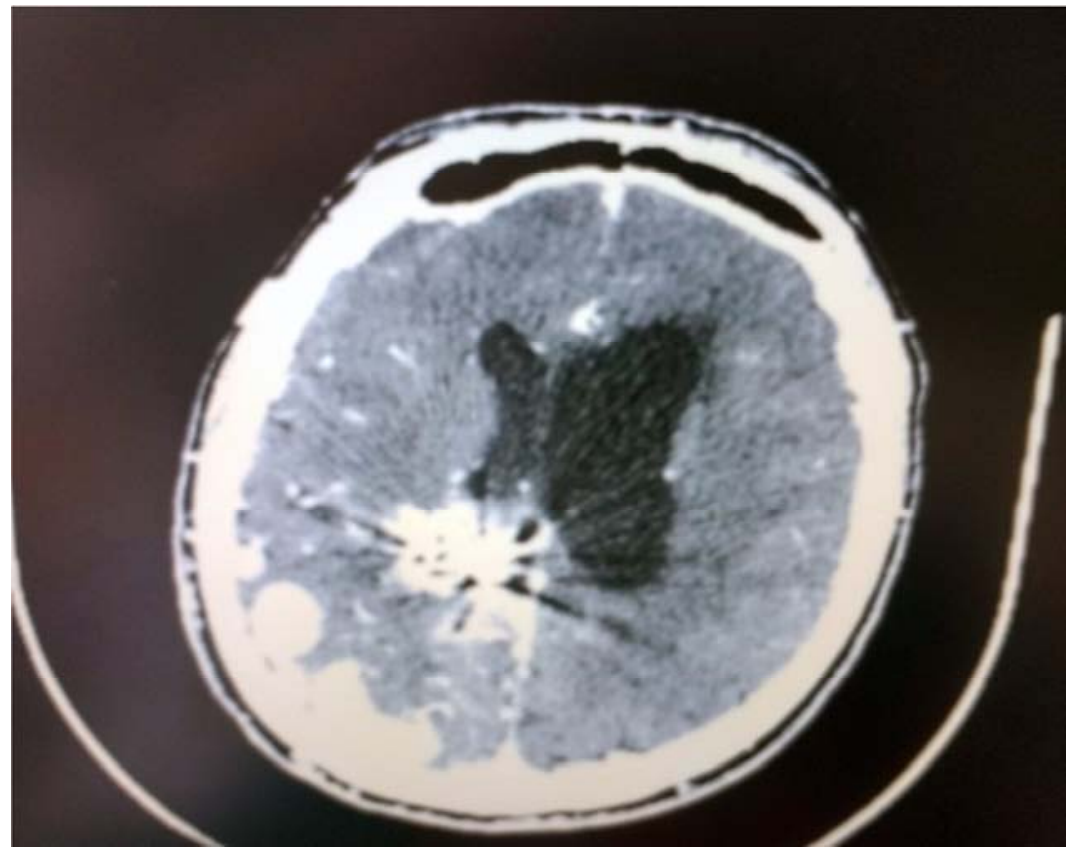

Figure 2. Preoperative axial CT scan depicting the partially embolized AVM, dilated cortical draining veins and hydrocephalus. 


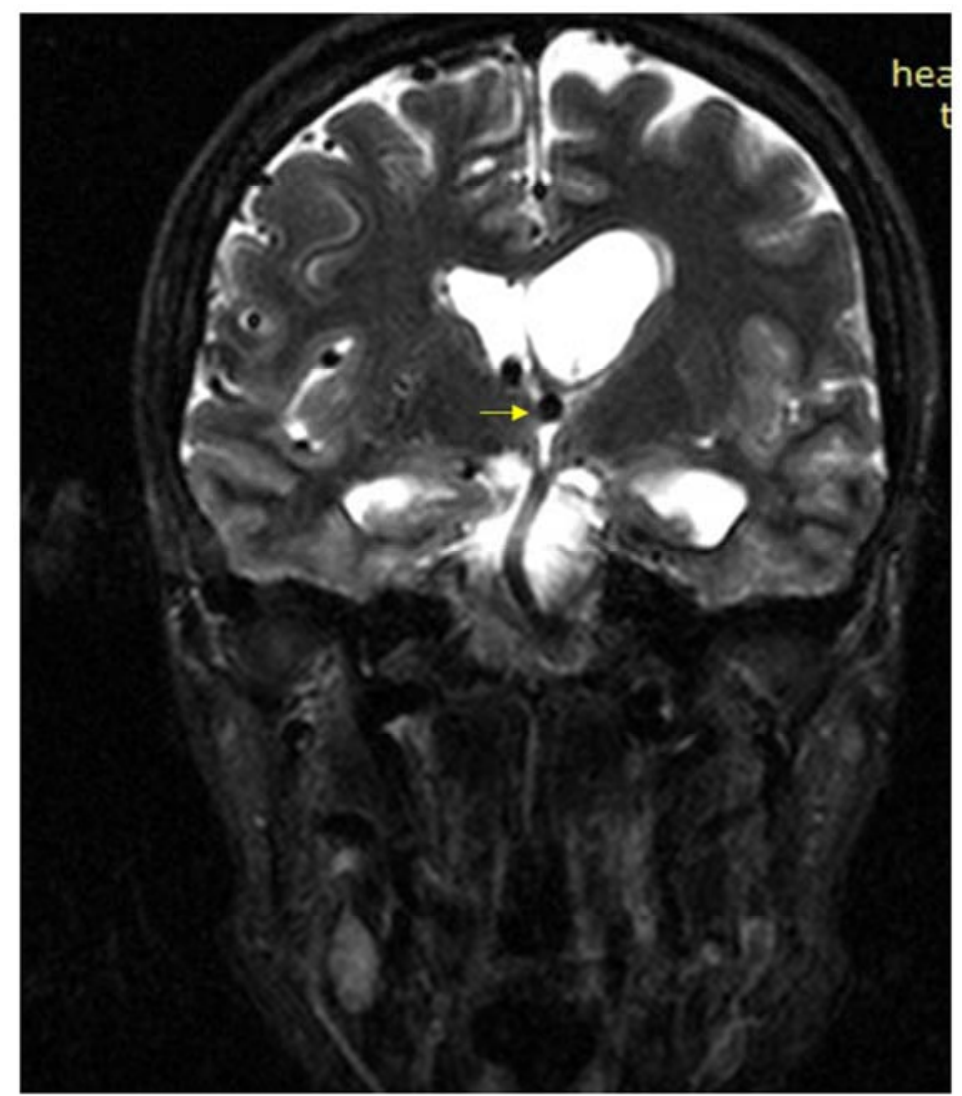

Figure 3. Preoperative coronal T2 MRI depicting obstructive hydrocephalus and dilated veins. Yellow arrow: left dilated internal cerebral vein causing obstructive hydrocephalus at the level of Monroe foramen.

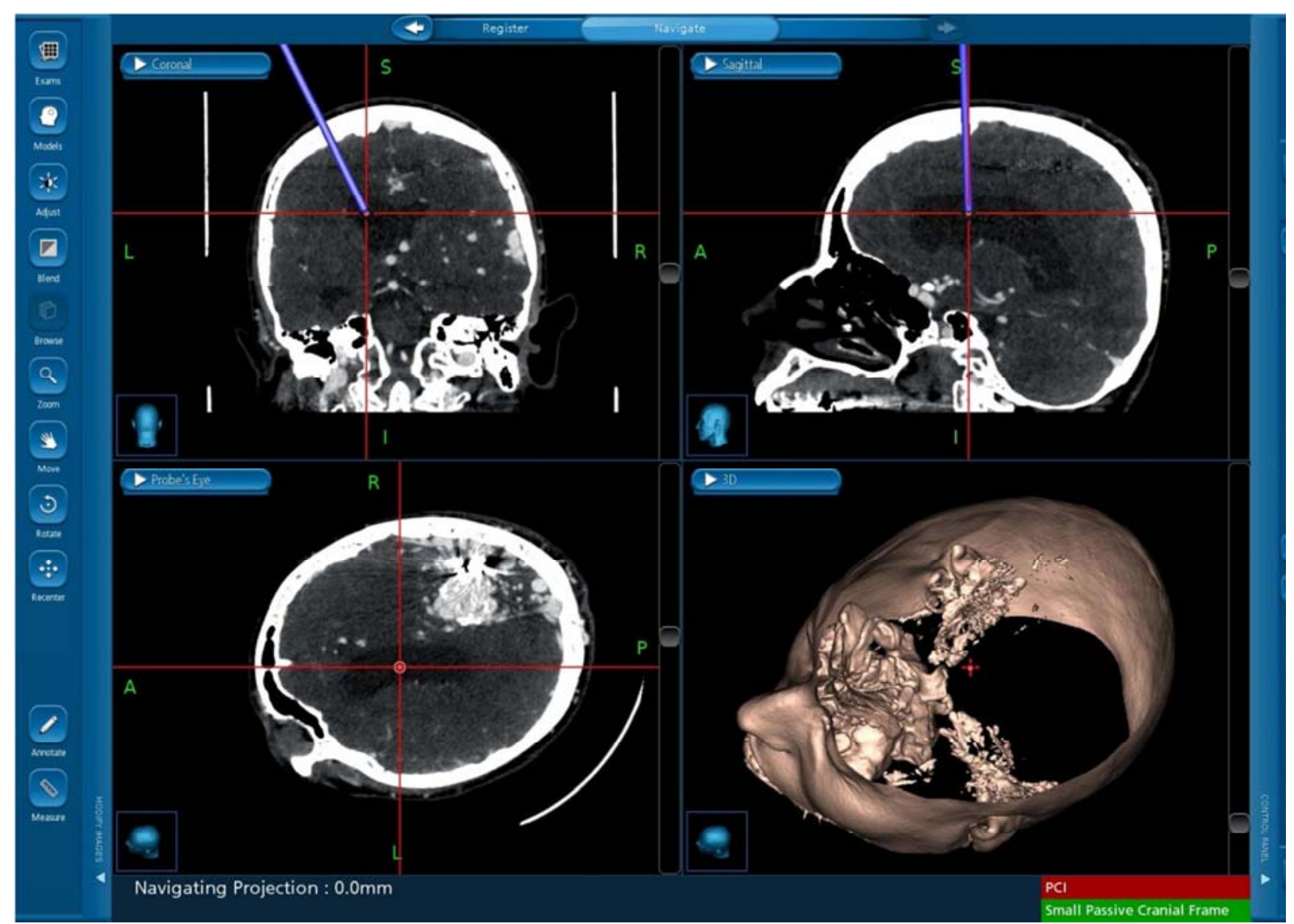

Figure 4. Intraoperative neuronavigation snapshot. 


\section{Results}

The application of neuronavigation resulted in safe and accurate placement of the ventricular catheter as it is verified by the postoperative MRI. (Figure 5)

Patient was examined at one month follow up and the hemiparesis and spasticity were improved. Evaluation was done with Modified Ashworth scale [7] (elbow 1+, wrist 1, finger 2, thumb, hamstring $1+$, quadriceps 2 , gastrocnemius 1 , soleus 1). Hemiparesis and gait were also improved.

Brain MRI depicted complete resolution of hydrocephalus. (Figure 5).

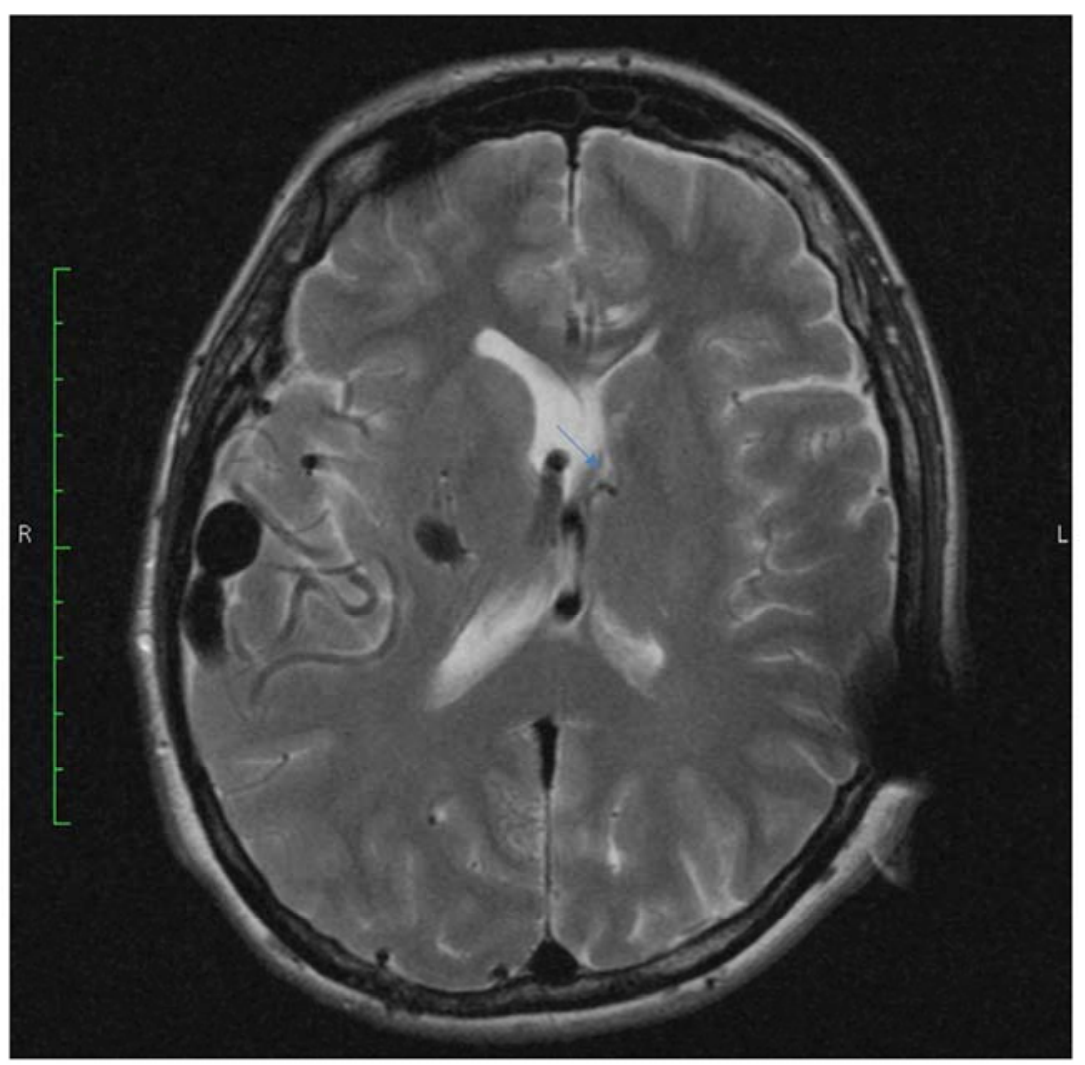

Figure 5. Postoperative T2 axial MRI depicting the ventricular catheter and the resolution of hydrocephalus. Blue arrow depicts the distal end of ventricular catheter inside the left ventricle.

\section{Conclusion}

Results depict that the ICP reduction caused by the shunt increased the functionality of the right motor cortex likely because of less venous obstruction and reduction of outflow resistance of the AVM. Furthermore, ventricular shunting in patients with obstructive hydrocephalus due to an AVM is a safe technique which should be applied with caution in respect with the anatomy of the AVM.

\section{References}

[1] Furkan Diren, Serra Sencer and Tayfun Hakan The Open Neuroimaging Journal, 2018, 12, 10-15 Case Report of an Obstructive Hydrocephalus Caused by an Unruptured Mesencephalic Arteriovenous Malformation in a Boy and a Review of Literature The Open Neuroimaging Journal, 2018, $12,10-15$.

[2] Lin F, Wu J, Zhao B, Tong X, Jin Z, Cao Y, Wang S Preoperative Functional Findings and Surgical Outcomes in
Patients with Motor Cortical Arteriovenous Malformation. World Neurosurg 2016 Jan; 85:273-81. doi: 10.1016/j.wneu.2015.10.002. Epub 2015 Oct 13.

[3] Paulsen RD, et al. Embolization of rolandic cortex arteriovenous malformations. Neurosurgery. 1999 Mar;44(3):479-84; discussion 484-6.

[4] Tucker A, et al. Endoscopic third ventriculostomy for hydrocephalus due to unruptured pineal AVM: case report and review of the literature J Neurol Surg A Cent Eur Neurosurg. 2013.

[5] Rossitti S. Pathophysiology of increased cerebrospinal fluid pressure associated to brain arteriovenous malformations: The hydraulic hypothesis Surg Neurol Int. 2013.

[6] Hoya K, Asai A, Sasaki T, Nagata K, Kimura K, Kirino T Expression of myosin heavy chain isoforms by smooth muscle cells in cerebral arteriovenous malformations Acta Neuropathol 2003 May; 105(5):455-61. Epub 2003 Jan 28.

[7] Pandyan AD, Johnson GR, Price CI, Curless RH, Barnes MP, Rodgers $\mathrm{H}$ A review of the properties and limitations of the Ashworth and modified Ashworth Scales as measures of spasticity Clin Rehabil 1999 Oct;13(5):373-83. 
[8] Hermnann EJ, Polemikos M, Heissler HE, Krauss JK Shunt Surgery in Idiopathic Intracranial Hypertension Aided by Electromagnetic Navigation Stereotact Funct Neurosurg 2017;95(1):26-33. doi: 10.1159/000453277. Epub 2017 Jan 14.

[9] Bembich S, Cont G, Bua J, Paviotti G, Demarini. S Cerebral Hemodynamics During Neonatal Cerebrospinal Fluid Removal Pediatric Neurol 2019 Jan 8. pii: S0887-8994(18)31063-4. doi: 10.1016/j.pediatrneurol.2019.01.005. [Epub ahead of print].

[10] Li M, Wang H, Ouyang Y, Yin M, Yin X Int J Surg Efficacy and safety of programmable shunt valves for hydrocephalus: A meta-analysis. 2017 Aug; 44:139-146. doi: 10.1016/j.ijsu.2017.06.078. Epub 2017 Jun 22.

[11] Charles CHAMPEAUX, Céline BOTELLA, Etienne LEFEVRE, Bertrand DEVAUX Obstructive Hydrocephalus Caused by an Unruptured Arteriovenous Malformation Successfully Treated by Endoscopic Third Ventriculostomy After Shunt Dysfunction Turk Neurosurg 28(3):500-504, 2018.

[12] Mascaros V, Eymann R, Marco J, Kiefer M The importance of hydrostatic valves in the treatment of adult chronic hydrocephalus Neurologia 2001 May;16(5):204-13.
[13] Tsuji A, Nozaki K A, Prospective and Retrospective Study of Cerebral AVM Treatment Strategies 1990-2014. Acta Neurochir Suppl 2016; 123:135-9 doi: 10.1007/978-3-319-29887-0_19.

[14] Müller A, Mould WA, Freeman WD, McBee N, Lane K, Dlugash R, Thompson R, Nekoovaght-Tak S, Madan V, Ali H, Stadnik A, Awad I, Hanley D, Ziai WC; CLEAR investigators The Incidence of Catheter Tract Hemorrhage and Catheter Placement Accuracy in the CLEAR III Trial Neurocrit Care. 2018 Aug; 29(1):23-32. doi: 10.1007/s12028-017-0492-6

[15] Khan NR, DeCuypere M, Vaughn BN, Klimo P, Image Guidance for Ventricular Shunt Surgery: An Analysis of Ventricular Size and Proximal Revision Rates Neurosurgery. 2019 Mar 1; 84(3):624-635. doi: 10.1093/neuros/nyy074

[16] Moftakhar P, Hauptman JS, Malkasian D, Martin NA Cerebral arteriovenous malformations. Part 2: physiology Neurosurg Focus. 2009 May; 26(5): E11. doi: 10.3171/2009.2. FOCUS09317 